\title{
HIGH-ENERGY ASTROPHYSICS
}

\section{(ASTROPHYSIQUE DES HAUTES ENERGIES)}

Report of Heetings, 8,9 and 10 August 1988

PRESIDENT : Catherine J. Cesarsky

INTRODUCTION

The Scientific sessions of Commission 48 at the XX General Assembly of the International Astronomical Union in Baltimore, USA, were focussed, on the one hand, on the most exciting recent event in the field : Supernova 1987A, and on the other hand on novel theoretical ideas. The first two sessions, held on August 8 and 9, were devoted to SN 1987A : the first to high energy observations, the second to theory; the second session ended with an animated general discussion of this fascinating object. The third-session, on August 10, was fully occupied by four extensive reviews, dealing with some recent developments in theoretical high energy astrophysics. Here is a list of the presentations given :

Session 1 : High energy observations of SN 1987A (chairman : F. Pacini)

Satellite results :

Y. Tanaka : (Institute of Space and Astronautical Science, Tokyo)

Results of the Ginga satellite.

J. Trümper : (Max-Planck Institut für Extraterrestrische Physik, Garching) The hard X-ray emission of SN 1987A (MIR-KVANT)

S.M. Matz : (N.R.L. Washington)

SMM gamma-ray observations of SN 1987A.

Balloon results :

W.S. Paciesas : (NASA/MSFC, Huntsville)

Balloon-borne hard $X$ ray experiment

P. Ubertini : (Istituto di Astrofisica Spaziale, Frascati)

Hard X-ray behaviour of SN 1987A

W.R. Cook : (Caltech, Pasadena)

An imaging observation of SN $1987 \mathrm{~A}$ at gamma-ray energies.

'B.J. Teegarden : (G.S.F.C., Washington)

GRIS observations of gamma radiation from SN 1987A : a preliminary report.

A.D. Zych : (University of California, Riverside)

Gamma-ray observation of SN 1987A above $1 \mathrm{MeV}$.

Ground observations :

E. Budding : (Carter National Observatory of New-Zealand, Wellington)

TeV gamma-rays from SN 1987A.

Session 2 : Supernova 1987A at high energies : theory

(Chairman : C. Cesarsky)

R. Mc Cray (JILA, Boulder)

$\mathrm{X}$-rays from supernova $1987 \mathrm{~A}$.

M. Salvati : (Osservatorio Astrofisico di Arcetri, Florence)

$\mathrm{X}$-rays from supernova $1987 \mathrm{~A}$ : beneath the radio active layers. 
T. Stanev : (Bartol Research Institute, Newark)

Acceleration of cosmic rays and production of high energy gamma-ray signals at SN 1987A

R.A. Chevalier : (University of Virginia, Charlottesville)

High energy phenomena related to SN 1987A.

General discussion, led by C. Cesarsky (SAp, Saclay).

Session 3 : Theoretical problems in contemporary high energy astrophysics. (Chairman : M. Rees).

R. Terlevitch : (Royal Greenwich Observatory, Hertsmonceux)

Star formation in Seyfert nuclei.

P. Meszaros : (Pennsylvania State University, University Park)

General relativistic effects in neutron stars.

J.P. Ostriker : (Princeton University, Princeton)

Superconducting strings and the high energy background.

R. Svensson : (Nordita, Copenhagen)

Pair plasmas in astrophysics.

A summary of the invited papers presented in all three sessions is presented below.

Session 1 : High.energy observations of SN 1987A

Experiments on board of three satellites have been following up the supernova since it first appeared at high energies in the summer of 1987 : the japanese Ginga satellite in X rays from 6 to $28 \mathrm{keV}$, the soviet-european complex of hard X-ray instruments on the MIR-KVANT module, and the american SMM satellite in gamma rays. Detailed accounts of the recent results of these three satellites were presented in Baltimore.

Y. Tanaka recalled that X-ray emission from SN 1987A has been observed continuously by the Ginga satellite since July 1987 , in the energy ${ }_{2}$ range 6-28 keV, with a set of proportional counters of effective area $4000 \mathrm{~cm}$. The shape of the hard X-ray spectrum, above $15 \mathrm{keV}$, is flat, perfectly compatible with the Compton tail expected from the degradation of ${ }^{56} \mathrm{Co}$-decay gamma rays. The spectrum of the soft component, at energies below $16 \mathrm{keV}$, is as expected from thermal bremsstrahlung from a gas of temperature in the range 10-12 keV. Apart from a burst in January 88 , the $X$-ray flux appears to have been declining very slowly between September 87 and July 88 ; the possibility of a steady flux cannot be rejected.

In January 1988 , a burst of $X$ rays was observed ; the intensity increased over two weeks, then declined over one month. The emissivity of the soft component rose by a factor of $\sim 3$; the spectrum changed as well. During the flare, an emission line was visible at $6.8 \pm 0.2 \mathrm{keV}$; it is interpreted as due to hydrogen-like or helium-like iron ions ; fluorescent emission would have occurred at lower energy $(6.4 \mathrm{keV})$.

J. Trümper reported the results of the MIR-KVANT observations. According to the author : It was a lucky coincidence that the KVANT module carrying the X-ray observatory "Röntgen" was launched a few weeks after SN 1987A was first observed. The docking to the MIR station was successfully completed in early April and the 
astronomical observations commenced on $\sim 3$ June 1988. The set of X-ray detectors includes a gas scintillation proportional counter (3-20 keV), a coded mask camera TTM (1 arcmin, 2-25 keV) and two Phoswich detectors (HEXE, 15-200 keV and PULSAR $\mathrm{X}-1$ 45-1000 keV).

Unfortunately, during June and July SN 1987A was observed only for rather short intervals (in total $3.25 \mathrm{~h}$ ), i.e. with limited sensitivity. The first long set of observations took place mid August 1987. During this period the hard X-ray flux of SN 1987A was first detected with the MIR-HEXE in the collimator rocking mode. In this mode source and background measurements are made simultaneously with two parts of the detector array. Almost all later measurements were performed by rocking the whole MIR station in order to allow the operation of the Pulsar X-1 instrument which has fixed collimators.

Between August 1987 and April 1988 a total of $\sim 96$ hours has been spent on SN 1987A. Figures 1 and 2 summarize the data obtained with the MIR-HEXE during this time.

After the failure of the TTM star tracker in April 1988 the Supernova has been observed for another $\sim 6$ hours (until mid August 1988). For this data only preliminary attitude information is available which comes from the MIR station. We prefer to derive source fluxes only after this attitude data has been calibrated. This calibration is in progress.

J. Trümper also commented on the comparison of MIR-KVANT data with other data : The Ginga data obtained at energies between 6 and $27.6 \mathrm{keV}$ is rather complementary to those of the MIR-KVANT in terms of energy coverage. The high energy part of the Ginga spectrum agrees reasonably well with the MIR-HEXE data in the overlapping energy range. The low energy component (6-16 keV) seen by. Ginga depicts rather large intensity fluctuations, and must be of different origin. Several balloon observations of SN 1987A have been made in fall 1987 and spring 1988 by the MSFC, the Frascati and the Caltech groups. The resulting hard X-ray fluxes confirm the data obtained with MIR-HEXE within the error limits which are quite large for the balloon experiments. Finally, J. Trümer reminded us that the hard X-ray emission of the SN 1987A had been predicted as a result of Comptonization of Cobalt 56 gamma rays in the expanding shell. However, the early GINGA and MIR-KVANT measurements showed a faster increase than the predicted one, suggesting a considerable mixing of the Cobalt 56 in the inner part of the shell. The rather flat light curve observed requires that the effect of the Cobalt 56 decay be largely compensated by a change in the Compton optical depth. However, this compensation cannot continue for a long time and one may expect that the hard X-ray flux will decrease later in 1988.

The gamma ray spectrometer on-board of the SMM satellite has been monitoring the ${ }^{56} \mathrm{Co}$ decay lines from SN1987A. The results were summarized by S.K. Matz : The Gamma-Ray Spectrometer (GRS) on NASA's Splar Maximum Mission satellite detected gamma ray line emission from the decay of ${ }^{56}$ Co in SN $1987 \mathrm{~A}$, beginning in 1987 August. Since the emission from the supernova has been continuously mlonitored with the GRS. The average fluxes from 1987 August 1 to 1988 April 6 are $(6.6 \pm 2.0) \times 10^{-4}$ and $(6.4 \pm 1.7) \times 10^{-4}$ photons $-\mathrm{cm}^{-2}-\mathrm{s}^{-1}$ for the 847 and $1238 \mathrm{keV}$ lines, respectively. The quoted errors represent a combination of statistical and systematic uncertainties. The best fit energies for the two lines during this period are $840 \pm 6$ and $1239 \pm 10 \mathrm{keV}$. There has been no marked increase in the intensity of either line since the end of 0ctober, and the data are consistent with a constant flux since August 1 . 


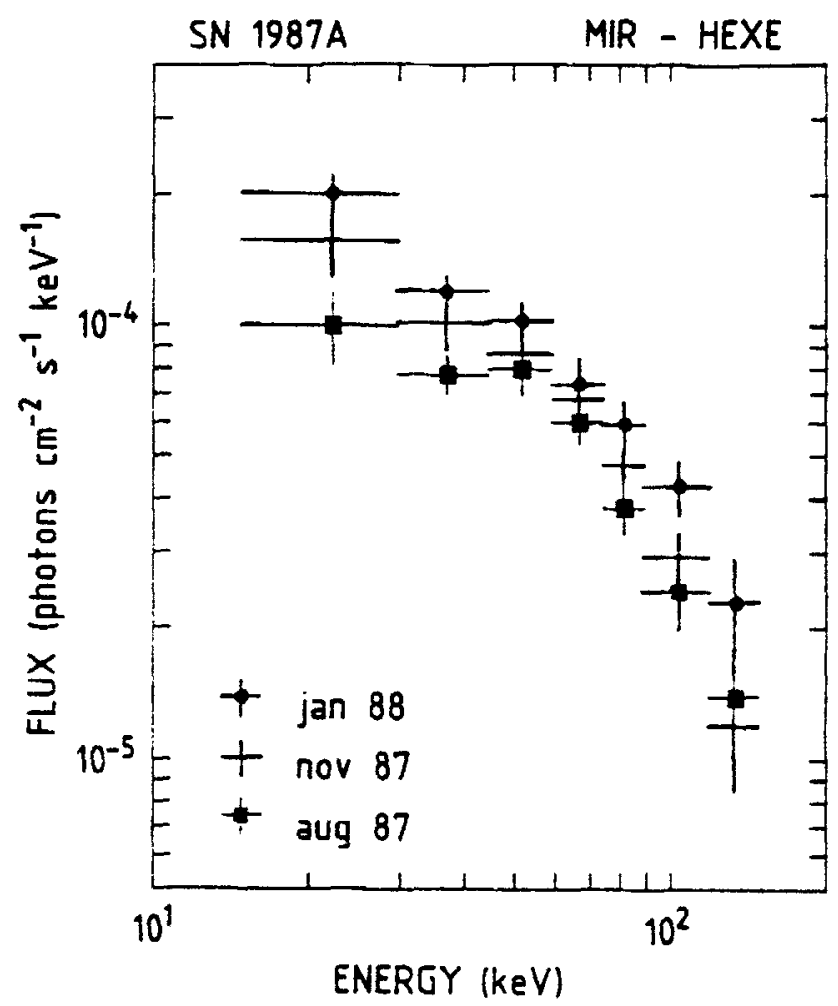

This was followed by a series of short presentations of balloon results. W. Paciesas spoke on behalf of the NASA-Lockheed collaboration : the region of the Large Magellanic Cloud containing SN 1987A was observed during a balloon-flight of a hard X-ray telescope on 8-10 April 1988. Significant continuum emission was detected in the $45-400 \mathrm{keV}$ range which is attributed to SN $1987 \mathrm{~A}$. Compared to an observation with the same instrument in October 1987, the source intensity decreased by approximately $50 \%$ while the spectral shape remained qualitatively the same. This may represent the first clear indication that the hard $\mathrm{X}$-ray emission is entering the declining phase expected as the ejecta become optically thin to the ${ }^{56}$ Co gamma-rays.

If there is a luminous $\left(\sim 10^{38} \mathrm{ergs} / \mathrm{s}\right) \mathrm{X}$-ray source inside SN 1987A, it should cause the bolometric light curve to become flat by $t=2.5$ years. Spectral evidence (at $\sim 20 \mathrm{keV}$ ) for a central compact source might also show up at roughly the same time. A pulsar will most likely be detected first ( $>4-5$ years) at optical wavelengths, or possibly at hard (>40 keV) $X$-rays if the envelope is fractured.

P. Ubertini presented the results of a hard X-ray experiment developed by an international consortium including groups from Italy, Australia, Germany, UK and USA : 


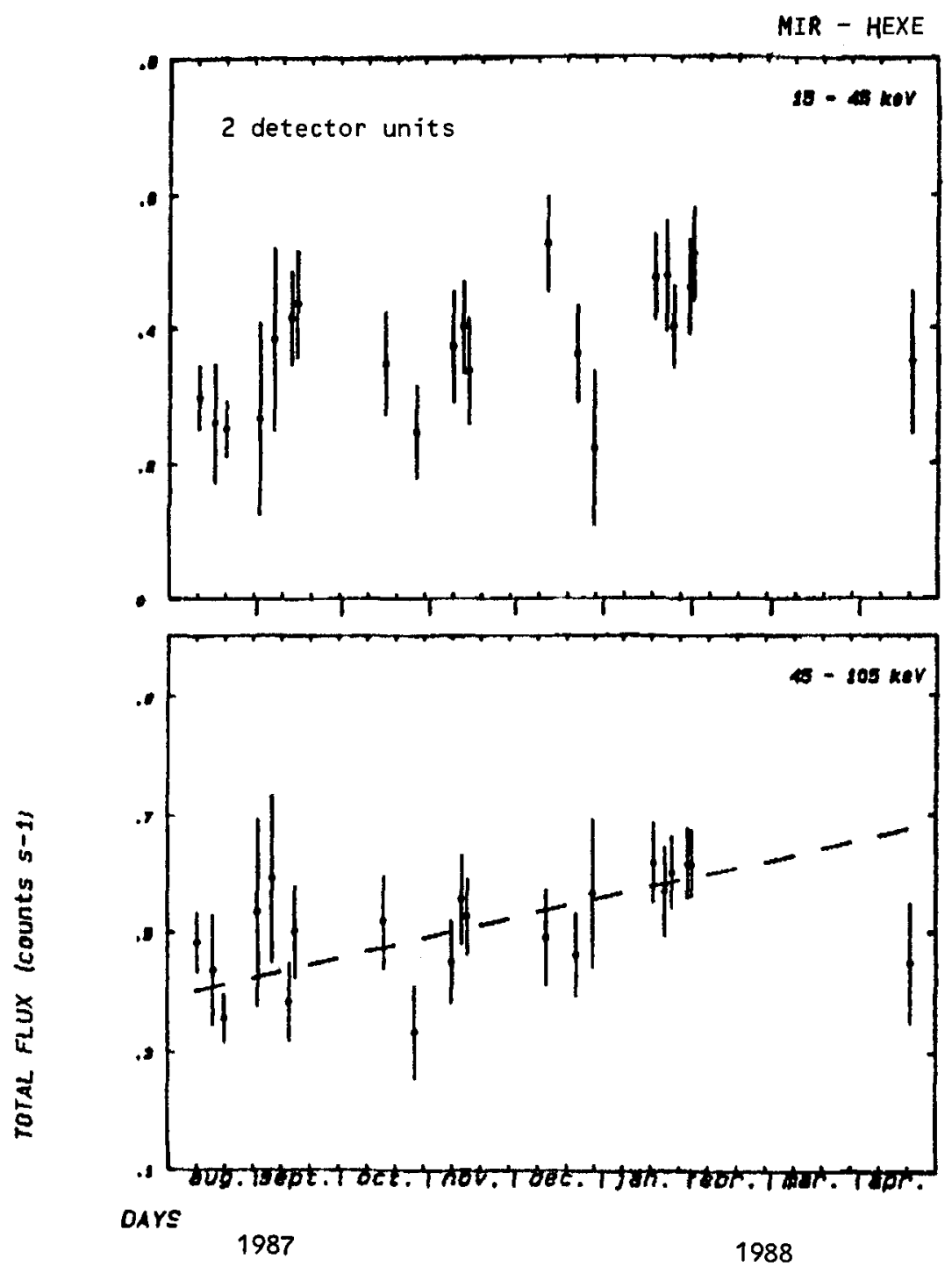


On April 5th 1988, the Supernova 1987A was observed between 00.00 and 09.55 UT with a hard $\mathrm{X}$-ray detector flown on a stratospheric balloon launched from Alice Springs, Australia. The balloon floated at an altitude corresponding to 3 to 4 mbar. The defector consisted of a 2.0 bar Xenon-filled MWPC with a sensitive area of $500 \mathrm{~cm}^{2}$ in the range $15-180 \mathrm{keV}$ and spectral resolution of $8 \%$ FWHM at $130 \mathrm{keV}$. The result of a preliminary analysis of the $\mathrm{X}$-ray data, shows a quite hard ( $\alpha-1,3$ in photons) spectrum over the range 20-160 keV, with an intensity of about $10^{-4} \mathrm{ph} / \mathrm{cm} \mathrm{s} \mathrm{keV}$ at $50 \mathrm{keV}$. The data obtained, if compared with earlier observations suggest an increase of the SN flux since last January.

$P$. Ubertini noted that, this increase of the flux is a real effect it may be an indication of an emerging low power pulsar.

He also mentioned results of a high energy gamma ray detector developed by the same consortium :

SN1987A has been observed with a spark chamber sensitive to high energy gamma-rays in the energy range $50-500 \mathrm{MeV}$. The observations were made during balloon flights on 19 April 1987 (day 55) and 5 April 1988 (day 406) from Alice Spring (Australia). Detailed analysis of the first flight showed no positive signal from SN $1987 \mathrm{~A}$ at the $3-\sigma$ upper limit of $2.9 \times 10^{-5}$ photons $/ \mathrm{cm}^{2} \mathrm{~s}$. This limit is consistent with an expanding mass of a few solar masses making the shell totally opaque to high energy gamma-rays at the time of the flight. Quick-look analysis of the second flight has placed a 3- $\sigma$ limit to the flux at $9 \times 10^{-4}$ photons $/ \mathrm{cm}^{2} \mathrm{~s}$. The supernova shell should have been transparent to gamma-rays by that fime. The limit therefore implies a cosmic-ray proton luminosity of $<5 \times 10^{41} \mathrm{ergs} / \mathrm{s}$ from the supernova. The team expects to further constrain this figure when the data analysis has been completed.

W.R. Cook showed observations of SN 1987A, taken by the Caltech imaging gamma-ray telescope (GRIP), a balloon-borne coded-aperture telescope operating in the range $10 \mathrm{keV}$ to $10 \mathrm{MeV}$. The instrument has been flown several times from Alice Springs, Australia, to observe SN 1987A. In May 87, the SN was not detected. In November 87 , it was seen at $4.9 \sigma$; in April 88, 8 t $5.4 \sigma$. The spectrum in the range $40-1300 \mathrm{keV}$ (including lines) is very flat ( $\alpha \mathrm{E}^{-0.9}$ ).

B.J. Teegarden presented surprising results obtained with the Gamma Ray Imaging Spectrometer (GRIS) developed by the GSFC in collaboration with the Bell and Sandia Laboratories. According to the author : the experiment consists of an array of seven large high-purity germanium detectors surrounded by a thick massive $\mathrm{NaI}$ anticoïncidence shield. The flight was launched from Alice Springs, Australia. On $30 \mathrm{Apr}$. and 1 May, 198811.3 hours of SN 1987A data were obtained. The experiment alternated every 20 minutes between target and background pointings. Background pointings were normally done by rgtating the experiment azimuth by $180^{\circ}$ while holding elevation constant. The -4 Co superngva line at $1238 \mathrm{kev}$ was detected at a flux level of $\left(8.1_{ \pm} 1.7\right) \times 10^{-4} \mathrm{photons} / \mathrm{cm}^{2}-\mathrm{sec}$. (The error quoted is statistical only; systematic errors remain to be evaluated). The statistical significance of this result is $4.9 \sigma$. The $1238 \mathrm{keV}$ line width is significantly larger than the instrumental line width. A preliminary estimate is 15-25 keV FWHM corresponding to a velocity range of $3600-6000 \mathrm{~km} / \mathrm{sec}$. Such large broadening may indicate that a substantial amount of the freshly synthetized material has mixed with faster moving material in the overlying mantle. The data also indicate an excess in the vicinity of the $847 \mathrm{keV}^{56} \mathrm{Co}$ line. Analysis is complicated by the presence of a strong instrumental background line at $844 \mathrm{keV}$. In addition a strong continuum signal was detected in the $120-700 \mathrm{keV}$ band. Further results will be forthcoming.

Finally, D. Zych described the University of California Compton Double 
Scatter Telescope, which features two arrays of scintillators of area $1 \mathrm{~m}^{2}$, one in plastic and the other in NaI. This imaging telescope is sensitive to gamma-rays from 1 to $30 \mathrm{MeV}$. The instrument was flown from Alice Springs on Apr11 15, 1988. The data analysis was on going at the time of the Baltimore IAU.

E. Budding made a brief presentation of the results of the JANZOS collaboration, which includes laboratories from Australia, New Zealand and Japan. The experiment, installed in the Black Birch Range in New Zealand, consists in one array of scintillators for the detection of UHE ( $>100 \mathrm{TeV}$ ) gamma rays, and a system of mirrors to detect Cerenkov light from VHE (>1TeV) gamma rays. The observations started in 0ctober 1987, and until now only upper limits are available, except for an interesting event : on January 14 and 15 , at a time coincident with the $X$-ray flare observed by GINGA, JANZoS detected an excess flux, of order $2.10^{-1} \mathrm{~cm}^{-2} \mathrm{~s}^{-1}$, of $\mathrm{TeV}$ gamma rays. The total energy emitted in this burst is comparable to that in the GINGA X ray burst $\left(\sim 10^{43}\right.$ ergs). The probabjlity of this excess to be a chance occurrence was estimated by the speaker at $10^{-4}$. This would be the first indication of acceleration of high energy cosmic rays by the compact object at the center of SN 1987A.

Session 2 : Supernova 1987A at high energies : theory.

The subject was introduced by $R$. Mc Cray, who gave a vivid summary of our current understanding of this problem. The interpretation of the hard (>15 $\mathrm{keV})$ $\mathrm{X}$-rays from SN 1987A is straightforward : gamma rays from ${ }^{56}$ Co decay are degraded by Comptonization to a typical emergent photon energy

$$
h v \sim \operatorname{mec}^{2} / \tau^{2}
$$

where $\tau_{c}$ is the Compton optical depth from the Co to the surface. Photoelectric absorption produces, a sharp low energy cut off in the emergent spectrum at approximately $20 \zeta^{1 / 4} \mathrm{keV}$, where $\zeta$ is the metallicity in solar units of the outer envelope. Detailed Monte-Carlo calculations show that the X-ray spectrum is a power-law, with photon spectral index $\alpha \sim 1.5$ when the X-rays first emerge. The spectrum becomes harder $(\alpha \rightarrow 1.0)$ with time. This general behavior of the spectrum is generic to the gamma ray down-Comptonization mechanism and is insensitive to the details of the supernova model. However, the time of first emergence of the - $X$-rays is sensitive to the model. Calculations based on models with stratified internal composition predicted that the $X$-rays would be detected initially at $t \sim$ 8 months after outburst. It is possible to account for the observed emergence of hard $X$-rays at $t \sim 4$ months by revising the interior model in such 5 way that the ${ }^{56} \mathrm{Co}$ is close to the surface. "Mixed" models, in which the "Co is stirred throughout the inner $5 \mathrm{M}$, can fit the $\mathrm{X}$-ray and gamma ray observations fairly well ; so cap "fractured" models, in which there are deep holes exposing part of the interior ${ }^{56} \mathrm{Co}$.

In contrast, there is no satisfactory interpretation of the soft (<15 keV) $\mathrm{X}$-rays observed by the Ginga satellite. They cannot be produced by down-Comptonization of gamma rays. Models have been constructed to fit the observed spectrum in which the X-rays come from shocks formed by the interaction of supernova ejecta with circumstellar matter. However, these models require circumstellar densities that are much greater than would be expected near the supernova progenitor star and that are in conflict with the early observations of radio emission from SN1987A. It has also been suggested that the soft X-rays are produced intrinsically by a neutron or synchrotron nebula at the center of the supernova. This interpretation would require thąt the supernova envelope has holes that are much more transparent (by factors $>10^{3}$ ) than the average envelope. If this explanation is correct, it is easier to account for the observed soft 
$\mathrm{X}$-ray variability and spectrum with an accretion source than with a Crab Nebula-like pulsar.

M. Salvati presented work done with R. Bandiera and F. Pacini on the possible contribution of a non-thermal nebula (plerion), produced by a rotating neutron star, to the high energy emission of SN1987A. Granted that the bolometric light curve of the SN and the hard $X$ ray spectrum are well accounted for by the presence of $0.07 \mathrm{M}$ of ${ }^{56} \mathrm{Co}$ after the explosion, there are still the problems of the early appearance of the hard radiation, and of the radiation below the photoelectric cut off. The authors argue that these characteristics of SN 1987A can be understood if two mechanisms are simultaneously at work. They should be relevant respectively, above and, below a photon energy $\sim 20 \mathrm{keV}$; besides being the location of the expected cut-off, this energy also corresponds to an observed spectral feature, indeed suggestive of a physical discontinuity.

A good candidate for the soft $X$-ray emission is synchrotron radiation from a non-thermal nebula, powered by a central neutron star. This can be detected if the envelope has undergone a process of early fragmentation, leading to the opening of transparent lines of sight towards the central region. Most of the hard X-ray flux can be provided by radioactivity, although an important contribution from the pulsar nebula cannot be ruled out. A possible fit to the spectrum observed at day 190 with these two components; is shown in figure 3 (where the dotted line represents the maximum, unperturbed plerion emission).

M. Salvati also emphasized the fact that, if the plerion is absent, the hard X ray flux of SN $1987 \mathrm{~A}$ will have decreased considerably by the end of 1988 .

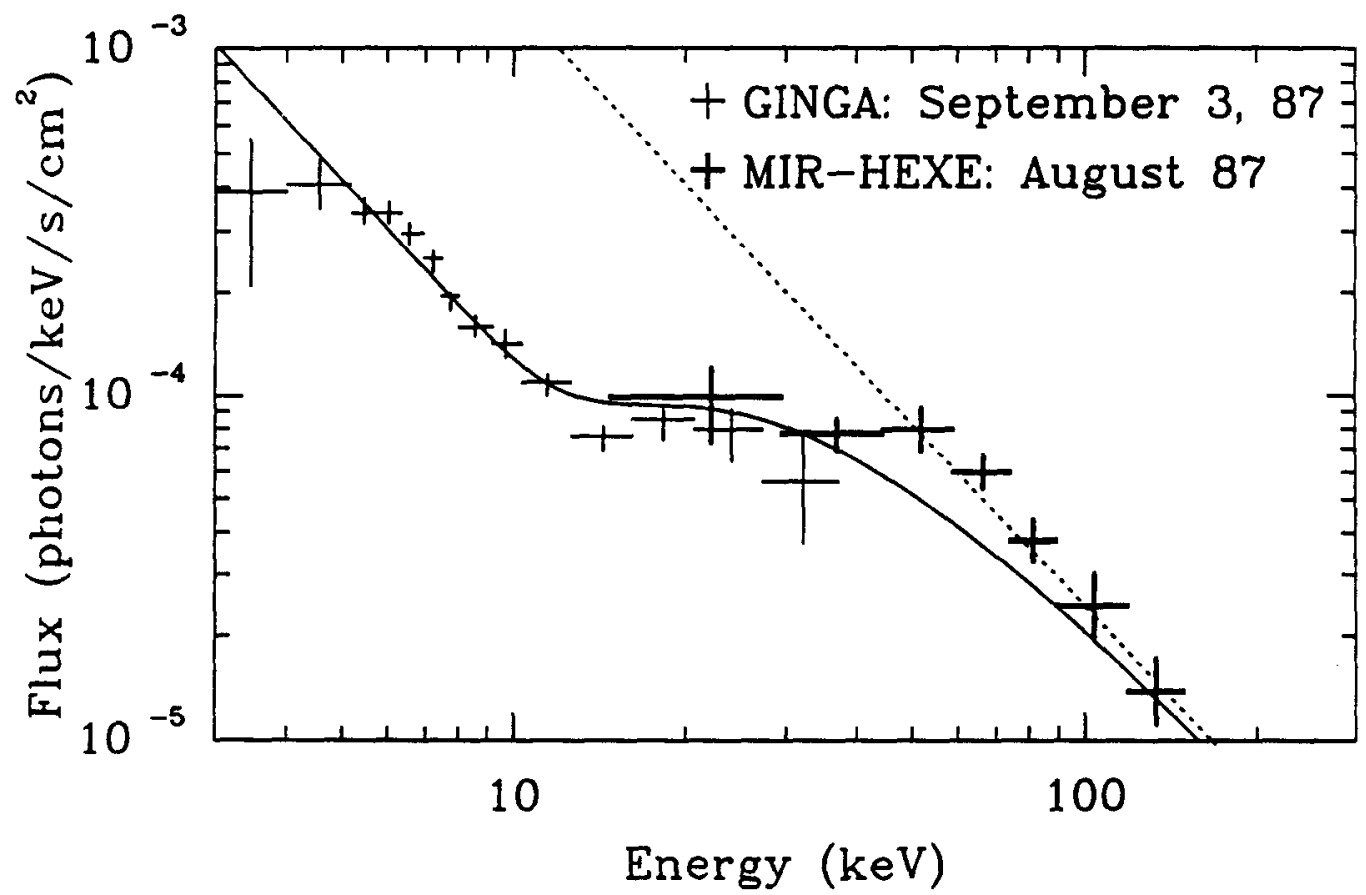

T. Stanev presented a study, made in collaboration with T. Gaisser, of the acceleration of cosmic rays and the production of high energy gamma-ray signals 
at SN 1987A. They developed a model which assumes that the pulsar radiation

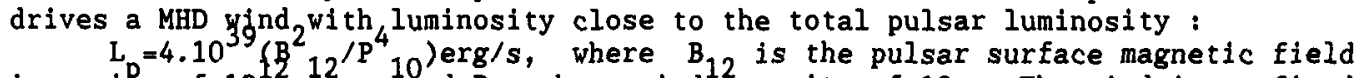
in units of $10^{12}$ gauss and $P_{10}$ the period in units of $10 \mathrm{~ms}$. The wind is confined inside the ejecta, a shock occurs at the radius where the wind ram pressure is balanced by the accumulated energy density of the wind, and charged particles are accelerated by first order Fermi acceleration at the shock. The acceleration of protons is only limited by the diffusion time away from the shock and for the minimum value of the diffusion coefficient the maximum achievable proton energy is $10^{1} \mathrm{eV}$ for $\mathrm{B}_{12}$ and $\mathrm{P}_{10}=1$.

Gamma rays are produced in the inelastic collisions of the protons with stellar material ; the exact amount energy to go into r-rays depends on the degree of mixing $h$ of shell material with the shocked wind containing the accelerated cosmic rays.

An observable photon signal will occur between characteristic times $t_{1}$ when the shell becomes optically thin to $>\mathrm{TeV}$ photons and $t_{2}$ when the shell becomes too dispersed for significant collision loss. $t$ occurs when the column density of the shell is roughly one radiation length, $\sim 1$ year after the explosion. One can imagine two extremes that bracket $t_{2}$ :

(a) If the accelerated protons are not contained in the shell the production of secondaries will cease when the column density of the shell becomes much less than one interaction length. This case was studied by Sato and his collaborators. (b) If the accelerated particles are confined indefinitely in the expanding remnant, $t_{2}$ will occur significantly later, after several years, when the expansion rate exceeds the interaction rate. In this case both the average time dependence and the magnitude of the signal will also depend on the nature and extent of mixing of the high energy particles with the expanding shell.

Light curves expected for confinement and for free propagation are shown in figure 4. The maximum $\gamma$-ray flux at Earth, corresponding to $\eta=1$, and full confinement of cosmic rays, was estimated through numerical calculations to be well agove the observational limit $-3.5 \times 10^{-11} \mathrm{~cm}^{-2} \mathrm{~s}^{-1}$ for cosmic ray luminosity $\mathrm{L}_{\mathrm{CR}}=10^{39} \mathrm{erg} / \mathrm{s}$ and integral proton spectral index $r_{\mathrm{p}}=1$.

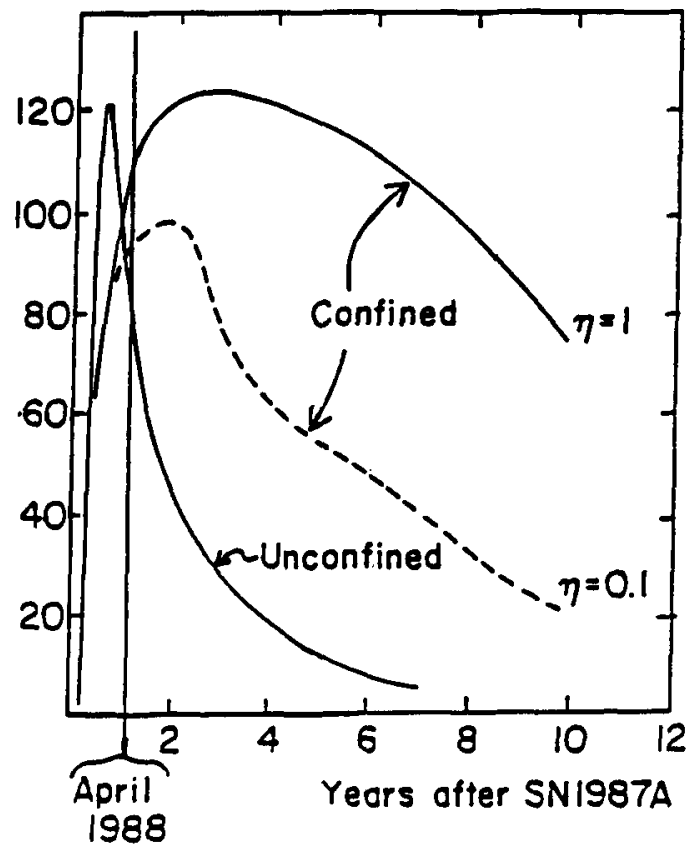


R. Chevalier dwelt on other high-energy phenomena related to the supernova :

1. When the shock front first breaks out of the atmosphere of the progenitor star, the photosphere is heated to a temperature $>300,000 \mathrm{~K}$. Because the opacity is dominated by electron scattering, the emitted photons originate deep within the star and a significant fraction of the radiation may be at soft $X$-ray energies. This pulse, lasting only minutes, was missed since it occured before the first optical observation. Scattering by interstellar dust (light echo effects) gives another chance to observe this radiation. However, the strong forward scattering of dust at X-ray energies implies that the echo would only be observable with current techniques during the first few months. Another effect of the hard photospheric radiation is ionization of the circumstellar medium. IUE observations of $\mathrm{NV}$ in a circumstellar shell suggest an effective temperature $>500,000 \mathrm{~K}$.

2. As the supernova shock front moves out into the surrounding medium, it can give rise to $\mathrm{X}$-ray emission and non thermal radio emission. The radio emission that was observed from SN 1987A during the first month was probably due to the shock interaction with the B3 I supergiant wind. The shock front energy X-rays have been detected with the Ginga Observatory, but the observed flux variability is a difficulty for a circumstellar interaction model. The shock front will eventually reach the dense matter that has been observed with the IUE, but this could take about 30 years. An estimate of the $1 \mathrm{GHz}$ radio flux at that time is 10Jy.

3. The neutrinos observed frgm SN 1987A suggest the formation of a neutron star and the ejection of $0.07 \mathrm{M}$ of ${ }^{51} \mathrm{Ni}$ indicates that there was not a large fall-back of matter onto the neutron star. The total luminosity of the supernova shows that an energetic pulsar is not present and suggests that neutron star accretion may become the dominant energy source. Estimates of the accretion rate in a supernova show that it is several orders of magnitudes larger than the Eddington limit rate. Under these conditions, an envelope can build up around the neutron star that prevents the escape of $X$-rays from close to the neutron star.

Session 3 : Theoretical problems in contemporary high energy astrophysics.

The session was opened by $R$. Terlevitch, who discussed his work (in collaboration with J. Melnick and G. Tenorio Tagle) on the relation between Seyfert nuclei and starbursts. He argued that nuclear starbursts can reproduce the observed properties of Seyfert nuclei. Models of starburst evolution show that after about 3 million years, the most massive stars become very hot and luminous ; they have been called "warmers" by the authors. When "warmers" begin to appear, the nebular spectrum changes in a very short time scale from a normal, low-excitation HII region, to a Seyfert or a Liner spectrum. The so called "broac line emission" regions observed in Seyfert type 1 nuclei may be young supernova remnants evolving in a high density interstellar medium : their observed properties (size, total energy, density, line widths) can be matched by such a model. The observed optical variability can also be understood in terms of sporadic explosions of type II supernovae. Thus the presence of a massive back hole may not be required.

P. Meszaros discussed general relativistic effects in neutron stars. The 
study of General Relativistic effects in neutron stars can lead to a determination of the radius of these objects, which when coupled with dynamic mass determinations allows one to reach conclusions concerning the equation of state, as well as concerning the energetics and spectral peculiarities of the emission. Observations and models of X-ray bursters lead to radi in the range

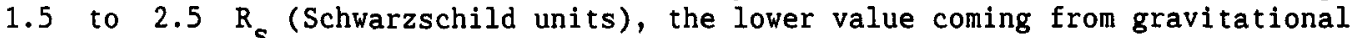
redshifts interpretations of absorption lines. For QPO sources, the magnetospheric beat frequency models can explain the very low upper limit to coherent pulsations if the radius is in the range $1.7-2.0 \mathrm{R}$. In accreting $X$-ray pulsars, accretion torque determinations in two objects showing cyclotron lines again suggest a value $<2-2.5 R_{s}$, while values $<2 R_{s}$ would help explain why systems which are suspected to be făn beam emittors mimic the spectral signatures of pencil beam emittors. Finally, gamma-ray bursters, if they are accreting magnetized neutron stars, require radii $<2.5 \mathrm{R}$ in order to explain the high incidence of 5-10 MeV bursts seen by SMM in a simple model where the low energy -absorption features are interpreted as straight cyclotron absorption. In the last three types of objects, it is the light bending which plays the crucial role, in QPOs suppressing the coherent pulsations from a low magnetic field broad cap, in X-ray pulsars in shifting the pulse phase of the emission from a small magnetic accretion colum, and in gamma-ray bursters in broadening the escape cone of high energy photons which avoid magnetic pair formation.

J.P. Ostriker presented recent work done at Princeton on superconducting strings and the high energy background. Reviews dealing with the properties of superconducting strings by Vilenkin (1985) and Thompson and Witten (1988) describe the physical basis for believing that superconducting strings may exist, the physical properties of such material, and the cosmological consequences of their existence.

For the present purpose it suffices to say that two parameters will determine $22^{\text {most }}$ of the astrophysical consequences : the mass per unit Iength (of order $\left.10^{22} \mathrm{gm} / \mathrm{cm}\right) \mu$ expressed as the dimensionless number $\mathrm{G \mu} / \mathrm{c}^{2} \sim 10^{-6}$ and the ratio of the electromagnetic output to the gravitational wave output, designated by $f$.

For cases of interest $f$ is of the order unity. One cosmic volume $(\mathrm{c} / \mathrm{H})^{3}$ will typically have one long string passing through it with a fractal distributions of irregularities down to very small scales, so that given the very high tenion, relativistic chaotic motions are induced which lead to self intersections and the generation of a network of smaller and smaller loops. These ultimately find equilibria stable against further fragmentation and then they oscillate losing energy via gravitational and electromagnetic radiation.

The dying old loops of length $L$ inject an energy into their surroundings equal to $f \mu \mathrm{Lc}^{2}$ in a manner quite analogous of a rapidly rotating pulsar. That is, the primary loss mechanism is via a Goldreich-Julian type relativistic MHD wind with a significant fraction of the output converted to high energy photons. Thus surrounding each radiation loop will be a giant version of the Crab nebula with quite analogous processes occuring within it for generation of X-rays, $\gamma-$ rays and cosmic rays. In addition the loops will excavate large bubbles in the baryon distribution capable of identification with observed aspects of large scale cosmic structure. Radio frequency emission is relatively suppressed due to synchrotro self absorption but Compton processes are significantlty enhanced due to strong interaction with the Cosmic Background (CBR).

At late epochs, the energy of the string will produce a high frequency distortion of the CBR through the Sunyaev Zeldovich effect whigh is significant : the induced $y$ parameter is approximately $y=2 \times 10^{-2} \mathrm{f}$ for $\left(\mathrm{G \mu} / \mathrm{c}^{2}\right)=10^{-6}$. Since most 
of this distortion is produced during a period $2>10^{2}$, when the universe is optically thick to electron scattering, the accompanying angular distortions are on scales greater than an arc minute and of amount $\Delta y_{\mathrm{rms}}=10^{-5}\left(\mathrm{f}=1 ; \mathrm{G \mu} / \mathrm{c}^{2}=10^{-6}\right)$, low enough to be under current obervational limits.

Finally, R. Svensson gave an able summary of studies on pair plasmas in astrophysics developped over the last six years. He distinguished two processes :

a) Thermal pair production

2 Wheg 1 electron temperature of plasma reaches approximately $m_{e} c^{2} / k=10^{9}-10^{10} k$, $i . e$. when the kinetic energies becomes of the same order as the electron rest mass energy then pair production may become important.

Detailed studies showed that for plasmas in pair equilibrium there exists a maximum temperature above which the annihilation of pairs cannot keep up with the pair production. The compactness :

$$
I=\frac{L}{\bar{R}} \frac{\sigma T}{m_{e} c^{3}}=3000\left(\frac{L}{\bar{L}_{\text {Edd }}^{-}}\right)\left(\begin{array}{l}
3 R_{s} \\
-\bar{R}^{-}
\end{array}\right)
$$

is the crucial parămeter, and in pair dominated plasmas the maximum temperature is a unique monotonically but slowly decreasing function of the compactness. For compactnesses $1<1<1000$, expected in compact objects, the maximum temperature is of the order $100-500 \mathrm{keV}$. Another important result is that an annihilation line is not expected from plasmas in pair equilibrium. At high temperatures other emission processes dominate the broad line and at smaller temperatures the Thomson depth is large enough to wipe out the line.

b) Non thermal Pair Production

Four main results emerge from detailed studies.

1. When the acceleration process puts most of the power into the highest energy particles, then in general most of the "primary" synchrotron and Compton radiation will be at the highest photon energies. If this energy exceeds $m_{c} c$ and the compactness is greater than unity, then photon-photon interactions will initiate a pair cascade that redistributes the gamma-ray luminosity into the UV-X-ray range thereby changing the spectral shape.

2. Characteristic spectral breaks are introduced with the main one occurring around $m_{e} c^{2}$.

3. The pair yield, PY, i.e. the fractin of the injectd power that ends up as pair rest mass, can reach values of $10 \%$.

4. The Thomsgn depth of cooled nonrelativistic pairs can reach values of $\tau_{\text {pair }} \approx(\text { PY } 1)^{192} \sim 20$.

He discussed then the application of these results to accretion flows.

A simple estimate of the maximum number of nonthermal pairs per accreted proton onto a black hole is obtained as follows : with an accretion efficiency of $10 \%$ there is about $0.1 \mathrm{~m} c^{2}=100 \mathrm{MeV}$ per proton available to make radiation and ultimately at most 100 pairs. A fraction, say $50 \%$, may end up as nonthermal radiation and a fraction PY $10 \%$ of this becomes pairs, i.e. 5 pairs or 10 particles per proton.

In two-temperature accretion flows (ion-torus) the presence of pairs affect the onset of Coulomb-cooling by the ions and the consequent collapse of the torus. 
In spherical accretion flows the amount of pairs for sub-Eddington accretion rates is negligible (as the $P Y<<1$ ). At Eddington rates the maximum pair-to-proton ratio derived above is reached, while at super-Eddington accretion rates the density is large enough for the pairs to annihilate on a inflow time scale reducing their number to negligible amounts.

Examples of application of these results to active galactic nuclei, to the cosmic X-ray background, and to gamma-ray bursts were given. 